\title{
ÍNDICE PARA A AVALIAÇÃO DE SEGMENTAÇÃO DE IMAGENS
}

\author{
Fernando Luís Dlugosz ${ }^{1}$, Nelson Carlos Rosot ${ }^{2}$, Maria Augusta Doetzer Rosot ${ }^{3}$, \\ Yeda Maria Malheiros de Oliveira ${ }^{3}$ \\ ${ }^{1}$ Eng. Florestal, M.Sc., Doutorando em Engenharia Florestal, UFPR, Curitiba, PR, Brasil - f.dlugosz@ gmail.com \\ ${ }^{2}$ Eng. Florestal, Depto. de Ciências Florestais, UFPR, Curitiba, PR, Brasil - ncrosot@ufpr.br \\ ${ }^{3}$ Eng $^{\mathrm{a}}$. Florestal, Dr ${ }^{\mathrm{a}}$., EMBRAPA Florestas, Colombo, PR, Brasil - augusta@ cnpf.embrapa.br - yeda@cnpf.embrapa.br
}

Recebido para publicação: 05/12/2005 - Aceito para publicação: 19/07/2008

\begin{abstract}
Resumo
O presente artigo descreve o desenvolvimento de um índice para avaliar quantitativamente resultados gerados por algoritmos segmentadores, tomando-se por base o Índice para Avaliação de Segmentação (IAVAS). As variáveis componentes do novo índice, denominado IAVAS mod $_{\text {, tiveram seus valores }}$ obtidos com a aplicação do método empírico de discrepância, que é dependente da existência de uma imagem de referência. Sobre essa imagem, também chamada segmentação-referência, efetua-se a comparação numérica relativa (em percentagem) dos resultados produzidos pelos valores de limiares aplicados ao algoritmo segmentador. A avaliação quantitativa dos resultados da segmentação envolve os seguintes parâmetros: número de polígonos, distância entre centroides, diferença em área, diferença em perímetro e faixa de coincidência. Métodos de avaliação quantitativa apresentam resultados mais consistentes em análises envolvendo segmentações, eliminando a subjetividade do analista, que ocorre quando se aplicam métodos qualitativos para a aceitação ou rejeição de valores de limiares.

Palavras-chave: Segmentação; avaliação quantitativa; índice IAVAS.
\end{abstract}

\begin{abstract}
An index for assessing the quality of image segmentation. This paper describes the development of an index for the quantitative assessment of segmentation algorithms, considering the Index for Segmentation Assessment (IAVAS) as a basis. The composing variables for the new index, called IAVAS $_{\text {mod, }}$, were acquired by applying the discrepancy empirical method, which depends on an existing reference image. Over this imagery, also called reference map, numeric comparisons (in percent) are carried out in order to compare distinct threshold values used by the segmentation algorithm. The following parameters were used to compose the new index: number of polygons, distance between centroids, differences in area, differences in perimeter and, coinciding buffers around polygons. Quantitative assessments are more robust than qualitative ones for they eliminate the analyst's subjectiveness in rejecting or accepting threshold values.

Keywords: Segmentation; quantitative assessment; IAVAS index.
\end{abstract}

\section{INTRODUÇÃO}

O processo de segmentação representa, geralmente, o primeiro passo no sentido de preparar as imagens de satélite para uma futura classificação temática, passo este que pode determinar o eventual sucesso ou fracasso de uma análise. $\mathrm{O}$ ato de segmentar uma imagem consiste em agrupar pixels com características similares em termos tonais e texturais, formando regiões homogêneas. Essas regiões são correspondentes às feições da superfície terrestre e irão servir de base para a análise e cartografia temática.

A operação de segmentação pode ser efetuada de modo manual ou automático. A segmentação manual baseia-se no reconhecimento visual das feições representadas na imagem, por meio da comparação de diferenças apresentadas pelos elementos interpretativos, principalmente cor, tonalidade e textura. Posteriormente ao seu reconhecimento, as feições são digitalizadas via tela do monitor. Os processos automáticos de segmentação procuram simular o comportamento do fotointérprete ao 
reconhecer áreas homogêneas na imagem, baseando-se em suas propriedades espectrais e espaciais (SPRING, 1998).

O resultado obtido pelo modo manual não é único, pois diferentes intérpretes podem gerar resultados diferentes, ou um mesmo intérprete pode gerar diferentes resultados em tempos diferentes. A segmentação automática, pelo contrário - por se basear em critérios de reconhecimento de similaridades ou de diferenças mensuráveis entre regiões adjacentes -, apresenta resultados sempre iguais quando se repete o processo várias vezes com os mesmos valores para os critérios. Outra vantagem apresentada pelo modo automático em relação ao manual é a rapidez de processamento da imagem, que favorece a viabilidade das análises em imagens.

A segmentação automática, em geral, baseia-se nas características de descontinuidade e similaridade dos tons de cinza de uma imagem. O método que avalia a descontinuidade considera a mudança abrupta dos valores de cinza e o método por similaridade fundamenta-se na agregação de pixels em função da sua semelhança com os pixels vizinhos (GONZALES; WINTZ, 1987).

Antunes (2003) comenta que não existe um modelo formal para a segmentação, sendo esse processo essencialmente empírico e, em geral, ajustável aos diferentes tipos de imagens, com limiares definidos conforme a complexidade dos alvos investigados.

O uso da segmentação anterior à classificação de imagens supera as limitações apresentadas com o processo de análise pontual (pixels analisados de forma isolada), que se baseia unicamente em atributos espectrais (SPRING, 1998). Os elementos analisados e utilizados na classificação serão as regiões resultantes da aplicação do segmentador utilizado na definição do espaço de atributos da classificação (VENTURIERI; SANTOS, 1998).

Por meio do processo de segmentação, é possível obter-se, de maneira rápida, a discriminação e a delimitação de fragmentos. Adicionalmente, os polígonos gerados pela segmentação - convertidos para vetor - podem ser usados como amostras de treinamento em futuras classificações digitais e, ainda, em classificações visuais, por meio de simples atribuição de classes aos polígonos.

De acordo com Gonzáles; Woods (2000), a seleção de critérios de similaridade é dependente do problema em consideração e também do tipo de dados (imagens) disponíveis. Para Coutinho (1997), a definição de valores para os limiares na segmentação de imagens depende, principalmente, do conhecimento dos padrões de repartição espacial dos objetos de cada área e da definição da generalização cartográfica ideal, em função da escala cartográfica da abordagem realizada. Contempla uma etapa que exige maior atenção devido à inexistência de valores padronizados para as variáveis do algoritmo e, também, pode influenciar diretamente na acurácia dos resultados da segmentação.

Se os valores dos limiares forem muito baixos, o processo não atribuirá pixels às classes (fragmentação excessiva). Por outro lado, se forem muito altos, pixels representativos de diferentes classes serão incorretamente agrupados (NASCIMENTO; ALMEIDA FILHO, 1996).

Segundo Zhang (1996), os resultados da segmentação podem ser avaliados por dois métodos. O primeiro - definido como método analítico - permite examinar e avaliar diretamente os algoritmos através da análise de seus princípios, suposições, propriedades, exigências, utilidade, complexidade etc., não dependendo de experimentos e podendo ser aplicado sem que o algoritmo esteja completamente implementado. A aplicação desse método é independente da natureza ou objetivo da segmentação. O segundo é o método empírico, que permite examinar e avaliar um algoritmo de forma indireta, através da aplicação de imagens-teste e medição da qualidade do resultado da segmentação. Esse método pode ser dividido em dois tipos: de discrepância e de qualidade.

De acordo com Lucca (1998) e Oliveira (2002), os métodos empíricos de qualidade permitem examinar e avaliar o desempenho de algoritmos julgando-se simplesmente a qualidade da segmentação final obtida, sem nenhum conhecimento a priori da segmentação verdadeira (referência). Forma e uniformidade dos segmentos, além da diferença espectral entre as regiões extraídas pelo algoritmo, são algumas medidas de qualidade propostas nesse método (OLIVEIRA, 2002).

Os métodos empíricos de discrepância permitem analisar o desempenho de um algoritmo através da comparação da segmentação de uma imagem produzida por ele com uma segmentação de referência, sendo que ambas as segmentações são obtidas a partir do mesmo dado de entrada. No caso em que o dado de entrada é uma imagem real, a segmentação de referência é geralmente obtida através de uma segmentação manual da imagem de entrada baseada na interpretação visual. No caso de imagens sintéticas, a segmentação de referência é obtida diretamente do seu processo de geração (LUCCA, 1998). 
Neubert et al. (2006) compararam o resultado do algoritmo segmentador de vários softwares. A avaliação foi realizada por meio de comparação visual entre os segmentos gerados e as áreas de referência interpretadas visualmente. Também foi empregada uma avaliação quantitativa com base na referência, sendo analisada a geometria dos segmentos por meio de parâmetros de área, perímetro e forma.

Constata-se que, na maioria das pesquisas brasileiras que utilizaram a operação de segmentação de imagens, a definição dos melhores valores de limiares para o processo foi efetuada somente por avaliação qualitativa. $\mathrm{O}$ uso de métodos de avaliação quantitativa se justifica por eliminar a subjetividade do analista, que ocorre quando se aplicam métodos qualitativos para a aceitação ou rejeição de pares de limiares.

$\mathrm{O}$ presente artigo descreve o desenvolvimento de um índice para avaliar quantitativamente resultados gerados por algoritmos segmentadores, tomando-se por base o Índice para Avaliação de Segmentação (IAVAS) descrito por Oliveira (2002). O novo índice proposto, denominado IAVAS ${ }_{\text {mod, }}$, apresenta alterações na forma de aquisição dos valores de seus parâmetros componentes - obtidos em percentagem -, o que permite uma avaliação conjunta ou individual dos parâmetros envolvidos.

\section{MATERIAIS E MÉTODOS}

No presente experimento, utilizou-se uma subcena Ikonos, tendo como objetivo discriminar tipologias florestais presentes em remanescente de Floresta Ombrófila Mista. A imagem utilizada foi uma subcena Ikonos que se refere à Reserva Florestal da Embrapa, localizada no município de Caçador, entre as coordenadas geográficas $26^{\circ} 50^{\prime}$ e $26^{\circ} 55^{\prime}$ de latitude sul e $50^{\circ} 05^{\prime}$ e $51^{\circ} 00^{\prime}$ de longitude oeste, região centro-oeste do estado de Santa Catarina. O imóvel compreende uma área 1.157,48 hectares, apresentando cobertura vegetal constituída pela Floresta Ombrófila Mista (FOM), sendo considerada como um dos últimos remanescentes do ecossistema que ainda mostram características originais.

O segmentador utilizado foi o algoritmo de crescimento de regiões - disponível no software SPRING -, que efetua o processamento com base nos limiares de similaridade e área. A similaridade referente à proximidade radiométrica entre pixels, correspondendo ao valor da distância euclidiana mínima entre as médias das regiões, abaixo da qual duas regiões são consideradas similares e, então, agrupadas. O limiar de área é o número mínimo de pixels para que uma região seja individualizada, sendo, portanto, o tamanho mínimo que cada segmento deve possuir na operação de segmentação.

Para este trabalho, os limiares de similaridade foram definidos sistematicamente, enquanto que o limiar de área teve seus valores definidos a partir de um valor-teste que foi recebendo incrementos de modo não-sistemático, à medida que os resultados dos processos iam sendo obtidos. Ao todo foram testados 20 pares de limiares de similaridade e área.

A avaliação quantitativa foi obtida a partir da aplicação do método empírico de discrepância. A aplicação desse método é dependente da existência de uma segmentação-referência ("verdade de campo") que represente a identificação adequada dos alvos presentes na cena, levando-se em consideração os limites de resolução espacial e espectral. Podem ser considerados como segmentação-referência, o produto de interpretação visual de imagens, mapas ou uma restituição fotogramétrica, devendo esses materiais apresentar a priori uma maior confiabilidade que os resultados da segmentação automática.

As diferenças apresentadas entre as segmentações e a referência são comparadas numericamente

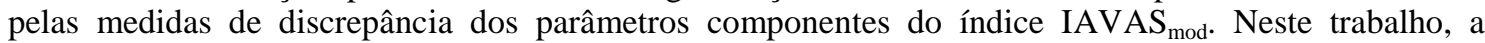
segmentação-referência utilizada foi o resultado da interpretação visual da mesma imagem Ikonos submetida aos testes de segmentação, que, após sua interpretação, passou pelo processo de digitalização manual em tela do monitor dos polígonos discriminados.

Os softwares de Sistemas de Informações Geográficas (SIG) - como, por exemplo, SPRING (INPE) e ArcView (ESRI) - apresentam ferramentas que auxiliam nas operações para a obtenção de valores dos parâmetros de discrepância necessários para o cálculo do IAVAS $\mathrm{mod}_{\text {mo }}$.

Tomando por exemplo o caso de a segmentação-referência apresentar-se no formato vetorial, inicialmente torna-se necessário converter os resultados do processo de segmentação - que são gerados em formato raster - também para o formato vetor, possibilitando, assim, calcular os valores dos parâmetros de análise.

A avaliação dos resultados da segmentação foi efetuada por amostragem sistemática, exceto para o parâmetro "número de polígonos", que é obtido diretamente. O emprego do método de amostragem proporciona uma redução de tempo e custo e torna mais dinâmico o processo de análise das segmentações. Foram selecionados na segmentação-referência e submetidos à análise os polígonos que se encontravam sob os pontos de cruzamento das linhas de um grid. 
Conforme a metodologia, o intervalo entre pontos do grid deve ser estabelecido de forma a atender a uma amostragem mínima de $10 \%$ do total de polígonos contidos na segmentação-referência. Para este estudo, o grid foi definido com intervalo entre pontos de $400 \mathrm{~m}$. Empregando-se ferramentas que permitam conhecer a intersecção entre dois temas, é possível conferir se a intensidade amostral está sendo atingida. Tendo-se obtido a amostragem adequada, os polígonos selecionados devem ser armazenados como um novo tema para facilitar os demais procedimentos.

A avaliação quantitativa dos resultados da segmentação envolve os seguintes parâmetros: número de polígonos, distância entre centroides, diferença em área, diferença em perímetro e faixa de coincidência. Sua descrição, bem como os passos para a obtenção de seus valores de análise, estão apresentados nos itens seguintes.

\section{1) Número de polígonos}

Esse parâmetro mede a discrepância entre a imagem segmentada e a de referência em relação à quantidade de objetos extraídos pelo algoritmo segmentador, permitindo, assim, uma verificação da ocorrência de segmentação excessiva ou insuficiente. Um bom conhecimento de campo e do objetivo do estudo torna-se importante para auxiliar na definição do número máximo de segmentos que será aceito.

De acordo com a metodologia, os valores de limiares que atenderam ao número máximo e mínimo preestabelecidos foram submetidos à análise dos demais parâmetros. A avaliação do parâmetro "número de polígonos" permite uma pré-identificação dos prováveis melhores limiares, não sendo, portanto, incluídos os valores desse parâmetro na equação do IAVAS ${ }_{\text {mod. }}$

\section{2) Distância entre centroides}

Esse parâmetro calcula a distância euclidiana entre os centroides de cada polígono da segmentação-referência e cada polígono da imagem segmentada. O centroide é uma entidade pontual que representa o centro de massa de determinado polígono. O cálculo da distância euclidiana é dado pela seguinte fórmula:

$$
d_{i j}=\sqrt{\left(x_{i}-x_{j}^{\prime}\right)^{2}+\left(y_{i}-y_{j}^{\prime}\right)^{2}}
$$

em que:

$i$ = número do polígono analisado da imagem referência;

$j$ = número do polígono analisado da segmentação;

$x_{i}$ e $y_{i}=$ coordenadas de $x$ e $y$ do ponto $i$;

$x_{j}^{\prime}$ e $y_{j}^{\prime}=$ coordenadas de $x$ e $y$ do ponto $j$.

Como a identificação dos polígonos equivalentes entre as imagens avaliadas é uma tarefa que apresenta grande dificuldade, optou-se pelo cálculo da distância euclidiana entre os polígonos da segmentação-referência - selecionados pela amostragem - e cada um dos " $n$ " polígonos gerados pelo valor de limiar sob avaliação, conforme ilustrado na figura 1.

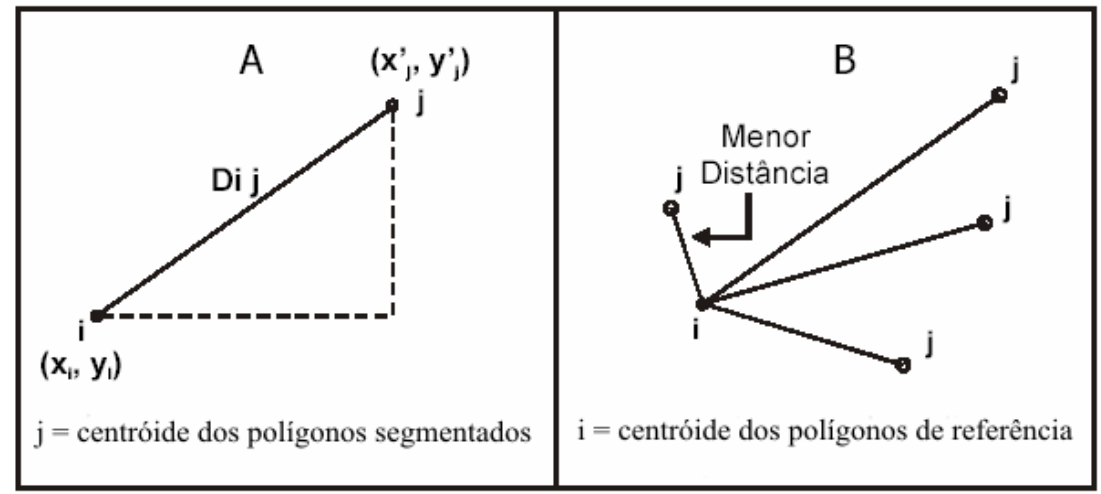

Fonte: Adaptado de Oliveira (2002)

Figura 1. (a) Distância euclidiana entre os centroides; (b) Polígono com menor distância euclidiana.

Figure 1. (a) Euclidean distance between centroids; (b) Polygon with the smallest Euclidean distance. 
Depois de calculada a distância das " $n$ " combinações, o polígono da segmentação que apresentou a menor distância em relação ao polígono $i$ avaliado da segmentação-referência foi aceito como equivalente e, posteriormente, o conjunto de polígonos selecionados de cada segmentação avaliada foi submetido à análise dos demais parâmetros.

O valor de discrepância para o parâmetro "Distância entre centroides" foi calculado após terem sido selecionados todos os menores valores das distâncias apresentadas pelas " $n$ " combinações. Em seguida, calculou-se a diferença percentual entre os valores do conjunto selecionado:

em que:

$$
V_{i}=\left(\frac{d_{i}-d_{\text {min }}}{\left(d_{\text {máx }}-d_{\text {min }}\right)}\right) \times 100
$$

$V_{i}=$ diferença percentual entre as menores distâncias selecionadas;

$d_{i}=$ valor da distância euclidiana de cada combinação selecionada do conjunto;

$d_{\text {min }}=$ menor valor de distância euclidiana do conjunto selecionado;

$d_{\text {máx }}=$ maior valor de distância euclidiana do conjunto selecionado.

Após a obtenção dos percentuais que uma dada distância entre centroides representa em relação à amplitude de distâncias observadas para todo o conjunto, foi calculada a média aritmética das menores diferenças percentuais selecionadas, passando esse valor a representar o parâmetro usado na comparação entre as segmentações. A média aritmética é dada por:

$$
\bar{d}_{e}=\frac{\sum_{i=1}^{n} V_{i}}{n}
$$

em que:

$\bar{d}_{e}=$ média da diferença percentual de distâncias euclidianas do conjunto analisado;

$V_{i}=$ valores da menor diferença percentual de distâncias euclidianas de cada polígono analisado;

$n$ = número de polígonos da segmentação-referência selecionados pela amostragem.

Quanto menor o valor apresentado por $\bar{d}_{e}$ mais semelhantes estão os polígonos da segmentação em relação aos da segmentação-referência.

\section{3) Diferença em área}

Para calcular esse parâmetro, foi necessário obter o valor de área de cada polígono-referência, assim como de cada polígono da segmentação selecionado no cálculo do parâmetro anteriormente descrito. As diferenças percentuais de área tiveram seus valores calculados de forma modular, utilizandose a equação:

$$
D A_{i}=\left|\frac{A R E F_{i}-A S E G_{i}}{A R E F_{i}}\right| \times 100
$$

em que:

$D A_{i}=$ diferença percentual de área entre cada polígono selecionado da segmentação e da segmentaçãoreferência;

$A R E F_{i}$ = área de cada polígono de referência analisado;

$A S E G_{i}$ = área de cada polígono da segmentação equivalente ao polígono de referência analisado.

Para o conhecimento do desempenho geral em cada valor de limiares da segmentação, foi calculada a média aritmética do conjunto analisado por meio da equação:

$$
\overline{D A}=\frac{\sum_{i=1}^{n} D A_{i}}{n}
$$


em que:

$\overline{D A}=$ média da diferença percentual de área entre a segmentação e a referência;

$D A_{i}=$ diferenças percentuais de área do conjunto analisado;

$n$ = número de polígonos da segmentação-referência selecionados pela amostragem.

Quanto menor o valor de $\overline{D A}$ apresentado pela segmentação, mais similares foram as áreas dos polígonos da segmentação e da segmentação-referência.

\section{4) Diferença em perímetro}

Os cálculos desse parâmetro foram efetuados de forma similar ao cálculo do parâmetro "Diferença em área", somente substituindo-se a variável "área" pela variável "perímetro", de acordo com as seguintes equações:

$$
D P_{i}=\left|\frac{P R E F_{i}-P S E G_{i}}{P R E F_{i}}\right| \times 100
$$

em que:

$D P_{i}=$ diferença percentual entre o perímetro de cada polígono selecionado da segmentação e da segmentação-referência;

$P R E F_{i}=$ perímetro de cada polígono de referência analisado;

$P S E G_{i}=$ perímetro de cada polígono da segmentação equivalente ao polígono de referência analisado.

$$
\overline{D P}=\frac{\sum_{i=1}^{n} D P_{i}}{n}
$$

em que:

$\overline{D P}=$ média das diferenças percentuais de perímetro entre a segmentação e a segmentação-referência;

$D P_{i}=$ diferenças percentuais de perímetro do conjunto analisado;

$n$ = número de polígonos da segmentação-referência selecionados pela amostragem.

Da mesma forma, a segmentação que apresentou o menor valor de $\overline{D P}$, em princípio, foi a mais adequada.

5) Faixa de coincidência

Os parâmetros de discrepância que avaliam separadamente o tamanho e a posição dos polígonos não asseguram uma total coerência nos resultados, pois podem ocorrer situações em que os segmentos estão rotacionados em seu centro de massa. Nesse caso, os valores calculados para tais parâmetros são considerados adequados, mas apresentam discrepâncias se avaliados quanto à forma de suas bordas, conforme ilustrado na figura 2.

O uso do parâmetro "Faixa de coincidência" permite avaliar espacialmente a relação entre os polígonos da segmentação e da segmentação-referência, analisando de maneira indireta, também, os parâmetros referentes ao tamanho e à posição. Isso se torna possível com a quantificação dos pixels da imagem segmentada que coincidem com os pixels da imagem de referência.

Durante a aquisição dos dados de referência por interpretação visual, o fator escala de trabalho pode ocasionar alguns erros, devido às limitações que o analista tem em definir o exato limite dos polígonos, podendo, assim, estar incluindo ou excluindo alguns pixels.

Para que esse fator não exerça influência na análise do resultado da segmentação, Oliveira (2002) recomenda a criação de uma faixa de tolerância de espessura igual à resolução da imagem utilizada, para dentro e para fora dos polígonos da imagem de referência. Os passos para a criação da faixa de tolerância foram os seguintes:

a) Gerar buffer nos polígonos da segmentação-referência ("verdade de campo"), criando tanto um anel interno quanto um externo (anel duplo), para uma distância igual à resolução da imagem utilizada, salvando os resultados dessa operação como um novo tema.

b) Unir os polígonos internos e externos gerados pelo buffer, formando um polígono único (anel simples). 


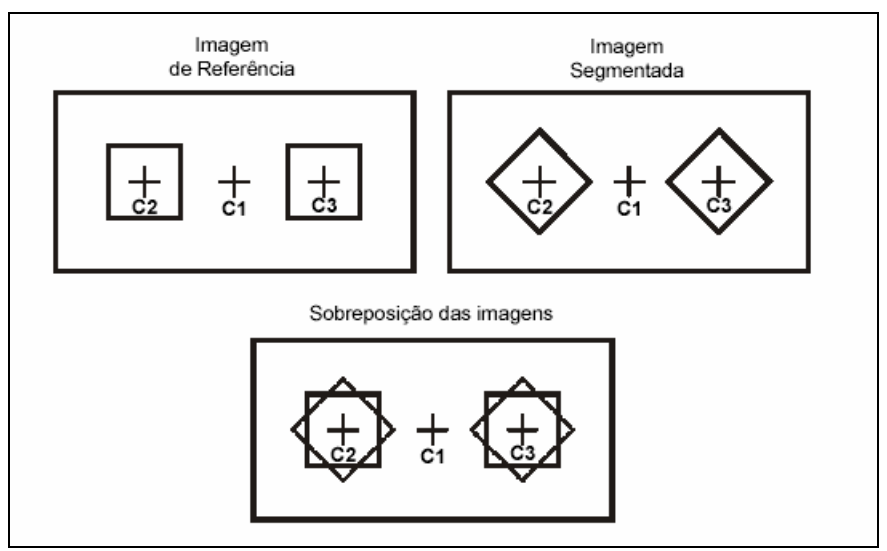

Fonte: Oliveira (2002)

Figura 2. Faixa de coincidência entre polígonos.

Figure 2. Coincident buffers between polygons.

A figura 3 ilustra os passos de criação da faixa de tolerância para uma imagem com resolução espacial de $4 \mathrm{~m}$.

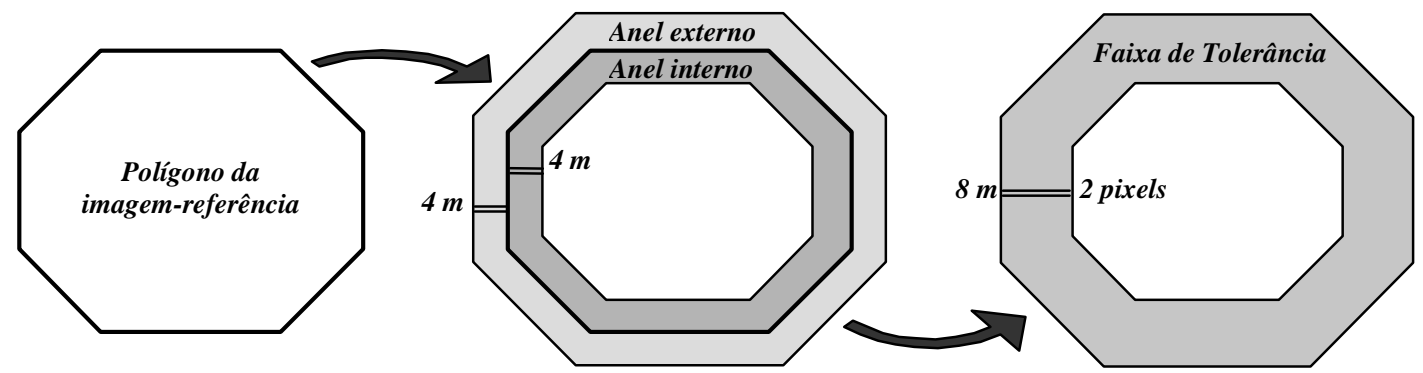

Figura 3. Criação da faixa de tolerância para uma imagem com resolução espacial de $4 \mathrm{~m}$.

Figure 3. Tolerance buffer for a four-meter-resolution image.

O cálculo do número de pixels das áreas de intersecção requer que os dados se encontrem em formato matricial (raster). Foi necessário, então, converter para esse formato o polígono da faixa de tolerância da segmentação-referência, assim como os polígonos da segmentação selecionados para as análises de discrepância. Como o polígono da faixa de tolerância apresentava-se em formato vazado (em forma de anel), a conversão foi realizada diretamente, observando-se que o tamanho da célula usado pela ferramenta fosse o mesmo valor da resolução espacial da imagem. No caso dos polígonos da segmentação (polígonos cheios), foi necessário convertê-los primeiramente para a feição "linha" e, sobre o tema de linhas, prosseguiu-se a conversão para o formato matricial.

Os valores comparativos do parâmetro "Faixa de coincidência" foram obtidos pelo cruzamento dos temas matriciais, realizando-se a somatória do número de pixels comuns entre cada segmentação e a referência. A partir desse valor da somatória e do número total de pixels apresentados pelos polígonos selecionados de cada segmentação, calculou-se o percentual de pixels espacialmente incorretos por meio da equação:

$$
F C=100-\left(\frac{n p_{\text {coinc }}}{n p_{\text {seg }}} \times 100\right)
$$

em que:

$F C=$ percentual de pixels espacialmente incorretos;

$n p_{\text {coinc }}=$ número de pixels coincidentes entre a segmentação e a segmentação-referência;

$n p_{\text {seg }}=$ número total de pixels dos polígonos da segmentação avaliada 
Nesse parâmetro, obtém-se em cada par de limiares um valor único para todos os segmentos gerados, efetuando-se, portanto, uma avaliação geral do desempenho na formação das regiões. A segmentação que apresentou o menor valor para $F C$ indica que menos pixels foram incorretamente associados a uma região e, consequentemente, mais semelhantes foram as regiões formadas em relação aos polígonos da segmentação-referência.

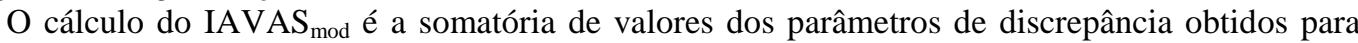
cada resultado do processo de segmentação, sendo dado pela equação:

$$
I A V A S_{\mathrm{mod}}=\bar{d}_{e}+\overline{D A}+\overline{D P}+F C
$$

Considerou-se como a melhor segmentação aquela que apresentou o menor valor no cálculo do índice IAVAS $\mathrm{S}_{\text {mod. }}$ Pode-se dizer que valores elevados são associados a grandes erros da segmentação, evidenciando um desempenho ineficiente do algoritmo segmentador.

\section{RESULTADOS E DISCUSSÃO}

Uma avaliação qualitativa da segmentação - sobrepondo-se a imagem a cada resultado proporcionado pelas combinações de pares de limiares -, possibilita conhecer de forma rápida o comportamento do algoritmo segmentador. No entanto essa avaliação pode não ser inteiramente confiável, devido à influência da subjetividade do analista durante a definição do melhor resultado.

Segundo Nascimento (1997), uma avaliação objetiva e quantitativa torna-se necessária para a escolha do melhor resultado do segmentador, visto que não há um valor ideal de limiar e eles variam de acordo com o material utilizado e o objetivo da segmentação.

O conhecimento do objetivo da segmentação é um aspecto relevante também para a aplicação efetiva dos métodos empíricos de discrepância, uma vez que ele baliza o resultado da segmentação manual que servirá de referência, assim como na definição das medidas de qualidades desejáveis acerca do resultado esperado.

A segmentação-referência utilizada foi resultado da interpretação visual da imagem Ikonos, em composição colorida Near Infrared+Green+Blue (NGB). A escolha dessa composição ocorreu devido à maior facilidade que essa composição proporcionava para o reconhecimento e discriminação das regiões (Figura 4). Conferiu-se a acuracidade do resultado da interpretação visual por meio de processo de reambulação, com avaliações em campo das regiões que durante a sua discriminação apresentaram dúvidas na definição dos limites.

De acordo com a metodologia utilizada no presente estudo para o desenvolvimento do índice IAVAS $_{\text {mod, }}$, a primeira etapa consistiu em analisar quais pares de limiares geraram uma quantidade de segmentos dentro de intervalo preestabelecido. O estudo propôs aceitar os resultados do algoritmo de segmentação por crescimento de regiões que geraram um número de segmentos igual ou até três vezes maior que o número de polígonos existente na segmentação-referência, de modo a evitar a ocorrência de segmentação insuficiente ou excessiva. Esse valor de três vezes foi definido após se considerarem as diferenças existentes entre o processo automático e o visual, no qual, por exemplo, áreas com sombra são agrupadas pelo intérprete às regiões adjacentes, enquanto que, no processo automático, elas são definidas como outra região.

$\mathrm{O}$ agrupamento de segmentos adjacentes na imagem torna-se facilitado ao intérprete quando este adquiriu em campo - na fase anterior à interpretação visual da imagem - informações sobre o comportamento das tipologias a serem mapeadas. Portanto, avaliou-se o resultado das segmentações que apresentaram um número mínimo de 427 segmentos (valor obtido na interpretação visual) e máximo 1281 segmentos.

Na figura 5 apresenta-se o comportamento do algoritmo por crescimento de regiões com relação ao número de segmentos formados para os limiares de similaridade e área testados.

Conforme se pode observar, o limiar de área foi o parâmetro que exerceu maior influência no número de polígonos gerados. Dos 20 pares de limiares testados, $15 \%$ apresentaram menos polígonos que o mapa-referência, 50\% apresentaram número excessivo de segmentos e 35\% atenderam o parâmetro de número de polígonos, sendo estes últimos submetidos à análise pelos outros parâmetros de discrepância. Os pares selecionados nessa fase foram: 30 e $2.000 ; 35$ e 1.200; 35 e 2.000; 40 e 1.200; 40 e 2.000; 45 e $1.200 ; 60$ e 600 . 


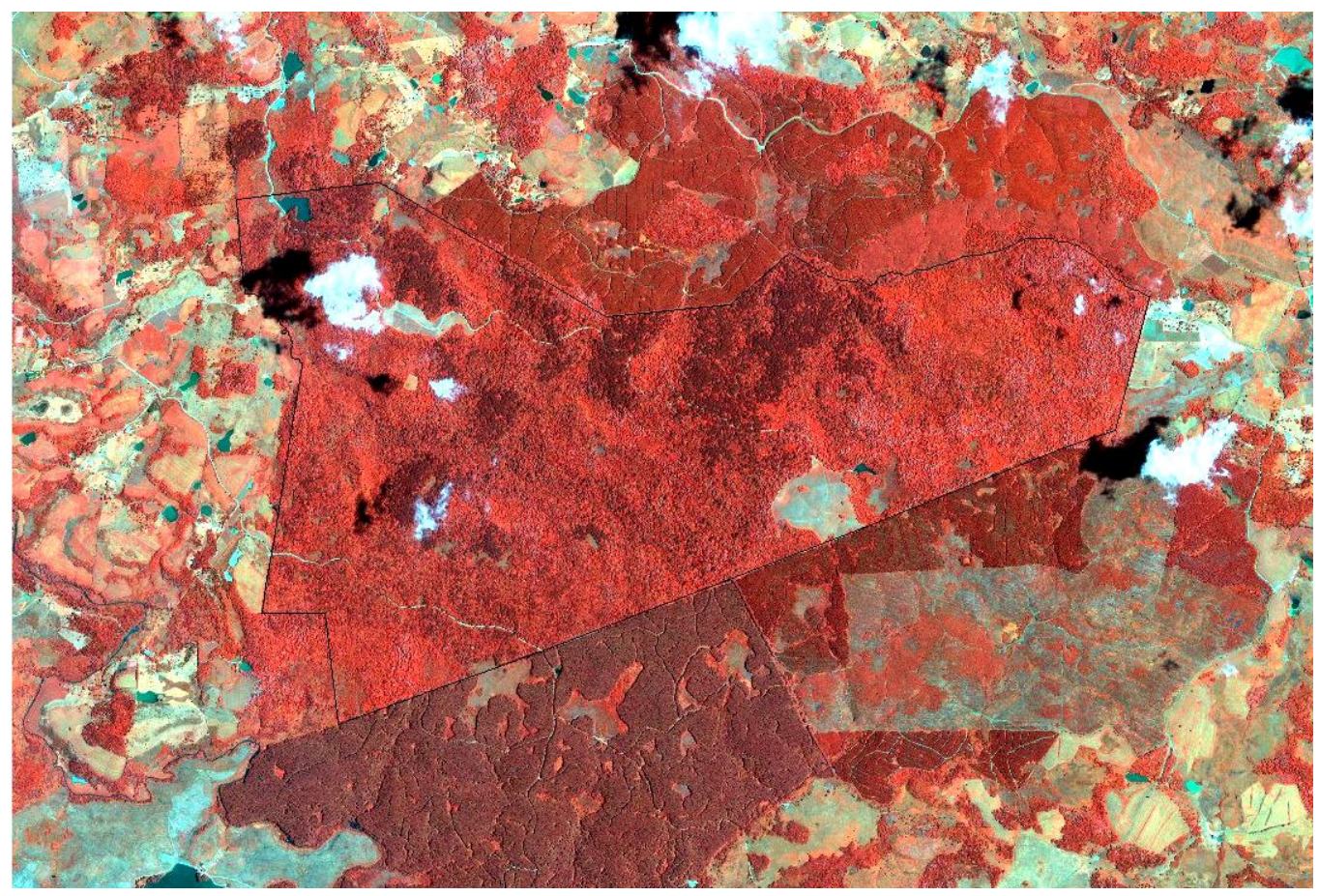

(a)

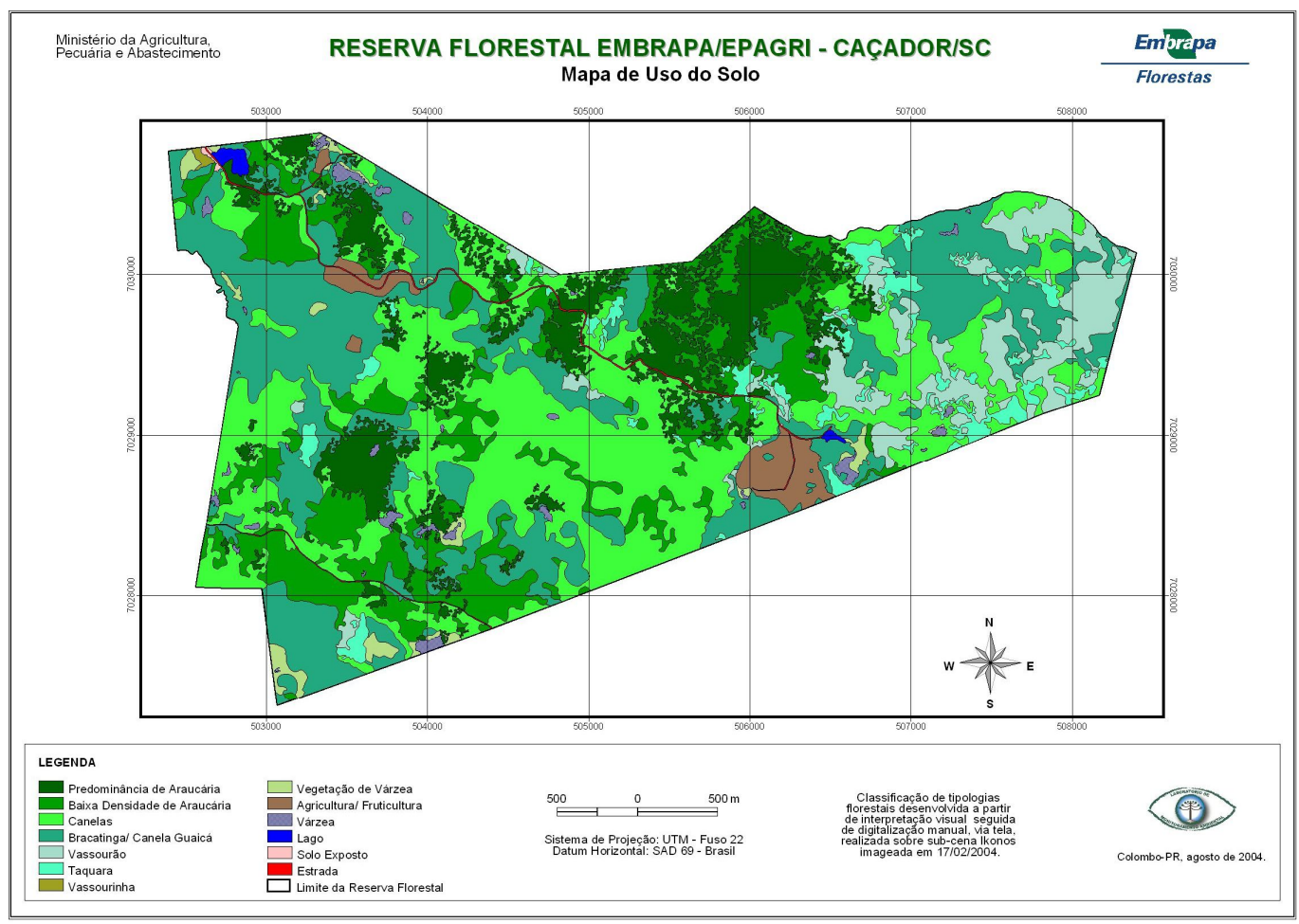

(b)

Figura 4. (a) Imagem Ikonos em composição NGB; (b) Segmentação-referência obtida por interpretação visual.

Figure 4. (a) Ikonos color-composite NGB; (b) Reference segmentation obtained by visual interpretation. 


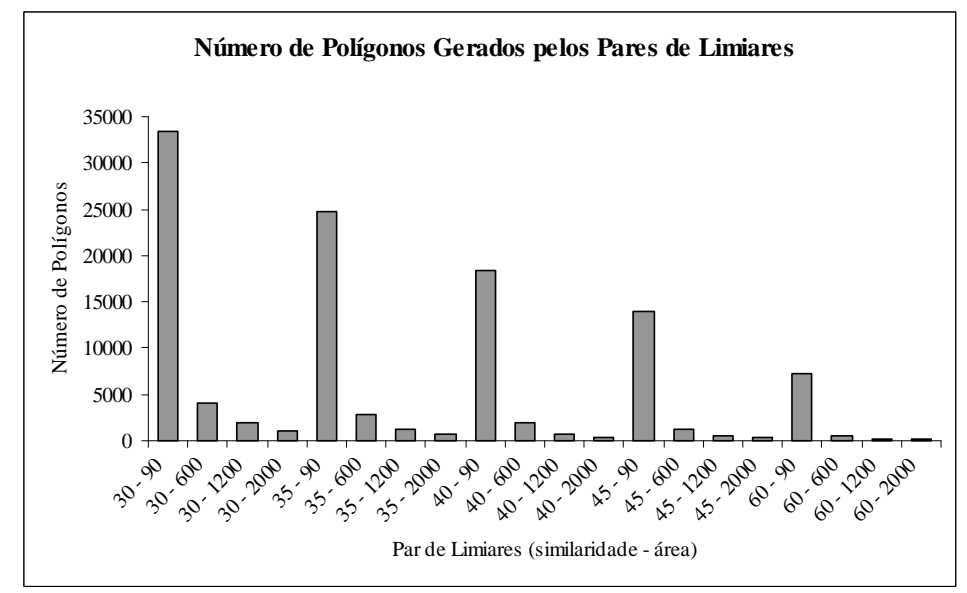

Figura 5. Número de polígonos gerados pelas combinações dos limiares da segmentação.

Figure 5. Number of polygons generated by each combination of the segmentation thresholds.

Na definição da amostragem da segmentação-referência, foram selecionados 51 polígonos que estavam sob o cruzamento das linhas do grid, correspondendo a 11,9\% dos polígonos discriminados. Depois de selecionados os polígonos da referência e definidos os equivalentes entre as segmentações, procedeu-se o cálculo da média percentual das menores distâncias entre centroides, dos parâmetros "diferença em área", "diferença em perímetro" e "faixa de coincidência" para cada segmentação. analisado.

A figura 6 apresenta uma representação gráfica do resultado de cada parâmetro de discrepância

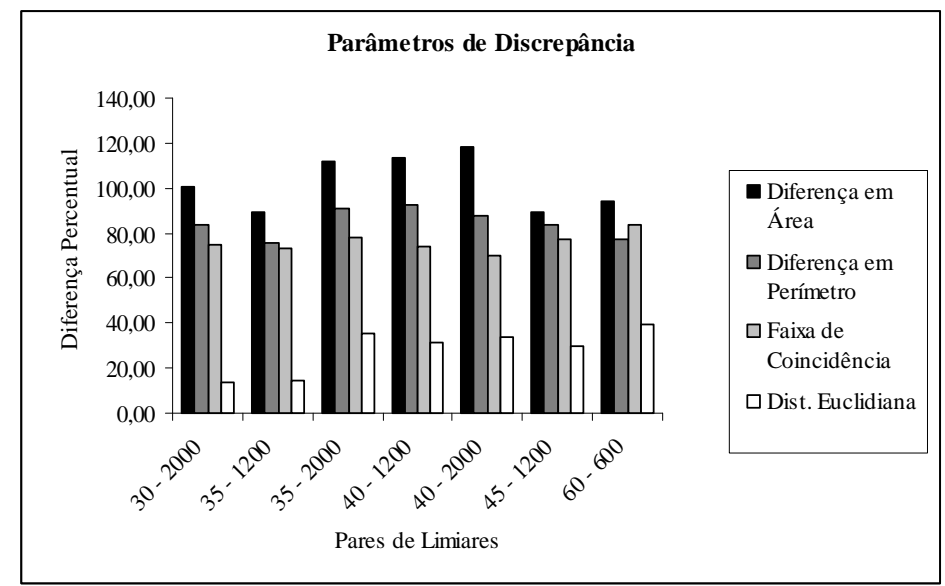

Figura 6. Comportamento dos parâmetros de discrepância conforme as combinações de limiares de similaridade e de área.

Figure 6. Discrepancy parameters behavior according to the combination of similarity and area thresholds.

Constata-se, que ao serem comparados os polígonos gerados pelo segmentador com os da segmentação-referência, a maior variação ocorreu sobre o parâmetro "diferença em área".

Os valores do índice $\mathrm{IAVAS}_{\text {mod }}$ alcançados pelos pares de limiares analisados foram ordenados em um ranking, permitindo, dessa forma, conhecer o limiar que apresentou a melhor performance de segmentação. A síntese dos resultados do índice IAVAS $_{\text {mod }}$ para as combinações de limiares testadas é apresentada na tabela 1 . 
Tabela 1. Síntese dos resultados da análise quantitativa da segmentação.

Table 1. Quantitative results of the segmentation analysis.

\begin{tabular}{lcccccc}
\hline $\begin{array}{l}\text { Par de } \\
\text { limiares }\end{array}$ & $\begin{array}{c}\text { Diferença } \\
\text { em área } \\
(\boldsymbol{\%})\end{array}$ & $\begin{array}{c}\text { Diferença em } \\
\text { perímetro } \\
(\boldsymbol{\%})\end{array}$ & $\begin{array}{c}\text { Distância } \\
\text { euclidiana } \\
(\boldsymbol{\%})\end{array}$ & $\begin{array}{c}\text { Faixa de } \\
\text { coincidência } \\
(\boldsymbol{\%})\end{array}$ & IAVAS $_{\text {mod }}$ & Ranking \\
\hline $30-2000$ & 100,32 & 83,99 & 13,67 & 74,87 & 272,85 & $\mathbf{2}$ \\
$35-1200$ & 89,10 & 75,38 & 14,19 & 72,92 & 251,59 & $\mathbf{1}$ \\
$35-2000$ & 112,22 & 91,16 & 35,25 & 77,77 & 316,40 & $\mathbf{7}$ \\
$40-1200$ & 113,80 & 92,29 & 30,99 & 74,38 & 311,45 & $\mathbf{5}$ \\
$40-2000$ & 118,34 & 87,53 & 33,58 & 70,35 & 309,80 & $\mathbf{6}$ \\
$45-1200$ & 89,24 & 83,92 & 29,69 & 76,88 & 279,73 & $\mathbf{3}$ \\
$60-600$ & 93,84 & 77,49 & 39,40 & 83,74 & 294,47 & $\mathbf{4}$ \\
\hline
\end{tabular}

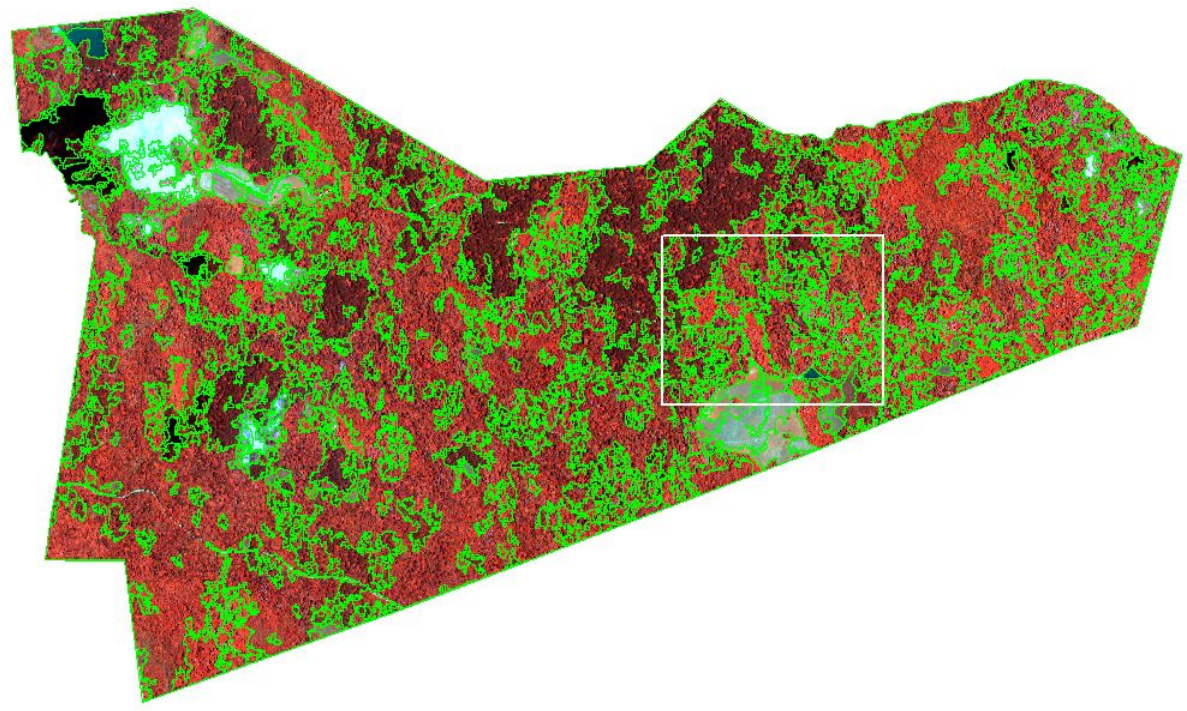

(a)

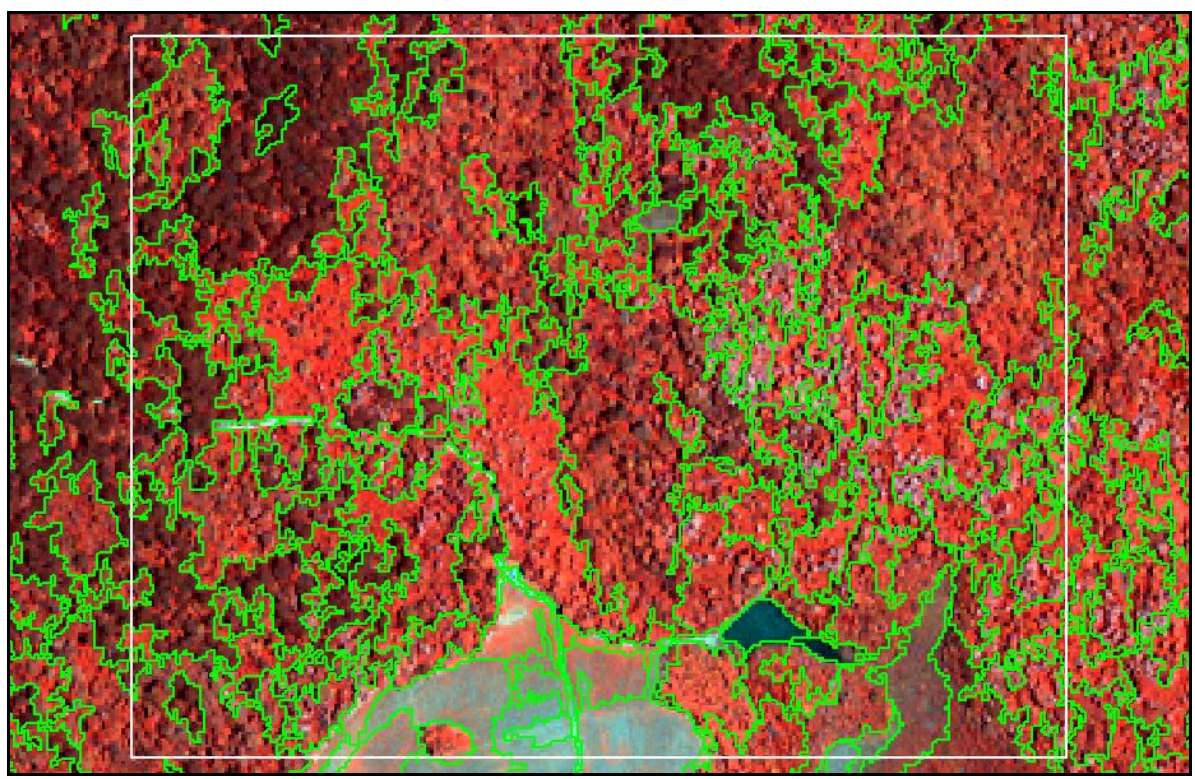

(b)

Figura 7. Resultado da segmentação com os limiares 35 e 1.200 para similaridade e área, respectivamente, e composição Ikonos NGB ao fundo: (a) área total; (b) detalhe ampliado.

Figure 7. Results of the segmentation for 35 and 1,200 similarity and area thresholds, respectively. Ikonos color composite in the background: (a) total area; (b) zoomed portion of the image. 
De acordo com os resultados obtidos com o método empírico de discrepância, a segmentação realizada com o par de limiares 35 para similaridade e 1.200 para área foi a que apresentou o menor valor no índice IAVAS $_{\text {mod, }}$, sendo considerada quantitativamente a segmentação mais coerente com a segmentação-referência. Na figura 7 encontra-se o resultado do par de limiares mais coerente com a referência.

Oliveira (2002) comenta que, em alguns casos, pequenas variações entre os limiares não alteram os valores dos parâmetros de discrepância, resultando em índices iguais. Em outras situações, diferentes combinações entre limiares fornecem resultados distintos para cada parâmetro, porém a soma deles é idêntica.

\section{CONCLUSÕES}

O presente trabalho procurou demonstrar que, apesar de ser mais complexa em sua aplicação, uma avaliação quantitativa é superior à qualitativa, por proporcionar resultados mais consistentes na análise de algoritmos de segmentação.

O índice $I_{A V A S}$ mod para a avaliação da segmentação de imagens permitiu comparações quantitativas para diferentes valores de limiares, eliminando, com isso, a subjetividade de uma avaliação qualitativa na definição do melhor resultado.

Com os valores dos parâmetros de discrepância apresentados em percentagem, tornou-se possível uma avaliação individual do comportamento de cada parâmetro para os pares testados, conhecendo sua influência no resultado obtido pelo segmentador.

A partir de valores de limiares que já se apresentem satisfatórios, recomenda-se testar intervalos menores para os parâmetros do algoritmo, como tentativa de alcançar valores mais acurados para o processo. Dessa forma, se conseguirá, também, elevar a acuracidade dos resultados da classificação da imagem, visto que são dois processos fortemente relacionados, considerando-se que os segmentos podem ser classificados a partir de características comuns de todos os pixels que os constituem.

\section{REFERÊNCIAS}

ANTUNES, A. F. B. Classificação de ambiente ciliar baseada em orientação a objeto em imagens de alta resolução espacial. 147 f. Tese (Doutorado em Ciências Geodésicas) - Setor de Ciências da Terra, Universidade Federal do Paraná, Curitiba, 2003.

COUTINHO, A. C. Segmentação e classificação de imagens Landsat-TM para o mapeamento dos usos da terra na região de Campinas, SP. 150 p. Dissertação (Mestrado em Ciências) - Instituto de Biociências, Universidade de São Paulo, São Paulo, 1997.

GONZALES, R. C; WINTZ, P. Digital image processing. Reading: Addison Wesley, 1987. 431 p.

GONZALES, R. C; WOODS, R. Processamento de imagens digitais. São Paulo: E. Blücher, 2000. 508 p.

LUCCA, E. V. D. Avaliação e comparação de algoritmos de segmentação de imagens de radar de abertura sintética.. 207 p. Dissertação (Mestrado em Sensoriamento Remoto). Instituto Nacional de Pesquisas Espaciais. São José dos Campos. 1998. Disponível em: <http://iris.sid.inpe.br:1912/rep/sid.inpe.br/deise/2000/ 04.27.16.28>. Acesso em: 30/11/2004.

NASCIMENTO, P. S. R. Avaliação de técnicas de segmentação e classificação por regiões em imagens Landsat-TM visando o mapeamento de unidades de paisagem na Amazônia. 120 p. Dissertação (Mestrado em Sensoriamento Remoto) - Instituto Nacional de Pesquisas Espaciais, São José dos Campos, 1997.

NASCIMENTO, P. S. R.; ALMEIDA FILHO, R. Utilização da técnica de segmentação em imagens TM/Landsat visando otimizar a técnica de interpretação visual. In: SIMPÓSIO BRASILEIRO DE SENSORIAMENTO REMOTO, 8., 1996, Salvador. Artigos... São José dos Campos: INPE/SELPER, 1996. 1 CD-ROM.

NEUBERT, M.; HEROLD, H.; MEINEL, G. Evaluation of remote sensing image segmentation quality - further results and concepts. 2006. Disponível em: <http://www.commission4.isprs.org/obia 
06/Papers/10_Adaption\%20and\%20further\%20development\%20II/OBIA2006_Neubert_Herold_Meinel. pdf>. Acesso em: 08/11/2007.

OLIVEIRA, J. C. Índice para avaliação de segmentação (IAVAS): uma aplicação em agricultura. 160 p. Dissertação (Mestrado em Sensoriamento Remoto) - Instituto Nacional de Pesquisas Espaciais. São José dos Campos, 2002. Disponível em: <http://iris.sid.inpe.br:1912/rep/sid.inpe.br/marciana /2003/03.05.15.25> Acesso em: 25/09/2004.

SPRING - Sistema de processamento de informações georreferenciadas. Processamento de imagens. São José dos Campos: NETGIS, 1998. v.2.

VENTURIERI, A.; SANTOS, J. R. dos. Técnicas de Classificação de Imagens para Análise de Cobertura Vegetal. In: ASSAD, E.D. e SANO, E.E. Sistema de Informações Geográficas. 2.ed., rev. e ampl.. Brasília, DF: Embrapa-SPI/Embrapa-CPAC, 1998. p. 351-371.

ZHANG, Y. J. A survey on evaluation methods for image segmentation. Pattern Recognition, Kidlington, v.29, n.8, 1996, p. 1335-1346. 